\title{
Uses and abuses of genetic engineering
}

\section{R Alexander}

\section{Ethical concerns remain central}

G netic engineering refers to the techniques whereby recombinant DNA, hybrid DNA made by artificially joining pieces of DNA from different sources, is produced and utilised. The term has gradually broadened out from this earlier more stringent definition to encompass virtually any process involving DNA manipulation. The applications of genetic engineering are now so widespread and well established within the biomedical sciences that it is difficult for younger investigators to envisage what research life was like in the era before genetic engineering. A quick skim of the articles in the current issue of the Journal of Immunology, a journal that ranges from clinical perspectives to molecular characterisation, reveals that out of 79 articles no less than 65 (82\%) utilised genetic engineering as an important component of their investigation. In the more molecular journals that figure would certainly be $100 \%$ and even in the most clinical journals genetic engineering utilisation is prominent. This helps to explain why biomedical research laboratories around the world now tend to look so similar: irrespective of the precise discipline involved, the widespread use of molecular biology imposes its own constraints upon architecture and, indeed, on the sociology of scientific communities.

The publication of the mouse genome sequence in late 2002 highlighted the enormous importance of the mouse as a model for human disease. ${ }^{1}$ Of the roughly 30000 genes present in both human and mouse, $99 \%$ of the human genes have homologues in the mouse genome (and vice versa). In practice what this means is that nearly all the genes that contribute to human disease can be studied in the mouse, although of course gene function is not necessarily identical in the two contexts. Deleting genes out of mice ("making knockouts" in laboratory jargon) and overexpressing genes in particular lineages ("making transgenics") has been with us for more than a decade. Today these earlier strategies are giving way to more sophisticated approaches, such as lineage-specific conditional knockouts in which a selected gene can be deleted in a particular cell lineage following its normal development. In "knock-in" strategies, point mutations can be introduced into a specific gene, enabling an exquisite level of specificity in structurefunction analysis. The old jibe that "all a knockout mouse would tell you is how a mouse copes without a particular gene product" has been addressed not only by such newer approaches, but also by the reconstitution of knockouts with a range of mutated versions of the deleted gene that again brings greater specificity into the story. In cancer studies a mouse can be engineered to develop a tumour, such as chronic myelogenous leukaemia caused by expression of the Bcr-Abl fusion protein (encoded by the Philadelphia chromosome in human chronic myelogenous leukaemia), and tumour regression then investigated following silencing of the transgene. ${ }^{2}$ By tagging tumour genes with a fluorescent probe the growth and remission of tumours can be readily assessed using whole body imaging. ${ }^{3}$ It is also now possible to introduce defined chromosomal rearrangements into the mouse genome by first genetically engineering them in embryonic stem cells. ${ }^{4}$ Other new technologies are enabling genomic DNA in bacterial artificial chromosomes to be directly modified and subcloned by a new approach known as "recombineering". 5

The rapid advances in the applications of genetic engineering to the mouse are mirrored to varying degrees by the speed at which the technology is transforming other research areas. As fig 1 illustrates, the applications of genetic engineering are myriad and the potential for positive use and potential misuse increases proportionally with the power and extent of the technology. The burgeoning biotechnology industry is to a large extent genetic engineering-driven and recombinant reagents are increasingly becoming a normal part of the pharmaceutical repertoire. In a typical application of genetic engineering to biotechnology, the US company Biogen has recently obtained licensing for its recombinant reagent Alefacept for the treatment of psoriasis, a T-cell mediated inflammatory disorder of the skin that affects about 100 million eople worldwide. Alefacept binds simultaneously to the CD2 antigen on T-cells and to a receptor expressed on NK cells thereby acting as a bridge to promote autologous killing of activated T-cells. Whether this mechanism explains Alefacept's clinical efficacy is a topic of active research. In a different kind of genetic engineering application, this time to identify novel pharmaceutical targets, the UK biotechnology company Polgen is using drosophila (fruit fly) to identify genes involved in cell cycle regulation. By systematically deleting the approximately 14000 or so genes in the drosophila genome using interfering RNA technology it is possible to work out which gene products control the cell cycle. Since around $40 \%$ of human genes are identifiable in the drosophila genome and genes that control the cell cycle are highly conserved in evolution, this approach is expected to reveal human gene products that could become drug targets in the treatment of cancer and other disorders involving uncontrolled cell proliferation. These two very different examples illustrate the breadth of application of genetic engineering in the biotechnology industry, ranging all the way from target identification and validation through to production of the pharmaceutical reagent itself.

The applications of genetic engineering to medicine may be broadly divided into two subdivisions, involving either diagnosis or treatment. Applications in diagnosis may be prenatal or postnatal. The use of genetically engineered probes in the prenatal diagnosis of disease using cultured amniotic cells or tissue obtained by chorionic villus tissue biopsy has greatly extended analytical techniques already being carried out using more traditional approaches. But in the case of preimplantation genetic diagnosis, the technique itself only became feasible by the use of genetic engineering, in this case by amplifying DNA sequences using the polymerase chain reaction (PCR). ${ }^{6}$ Like other prenatal diagnostic procedures, preimplantation genetic diagnosis is typically offered to parents who are already known carriers of genetically lethal mutations, including those involving familial predisposition to cancer. After in vitro fertilisation, one or two cells are removed from the very early embryo on day 3 at the 8-12 cell stage. Subsequently PCR amplification is carried out on DNA derived from a single cell and the DNA sequence is then investigated for the presence of the mutation. By amplifying several different sequences simultaneously (multiplex PCR), including the sequence known to contain the mutation and one or more containing polymorphic markers that are closely linked to that mutation, the possibility of misdiagnosis is decreased. The removal of two cells rather than one, although not essential, also enables 
duplicate assays to be carried out, providing additional confirmation. In the case of X-linked disorders in which no single cell methods are available to screen a specific mutant gene, sex determination can be carried out to ensure the implantation of female embryos only. Typically this is used for the prenatal diagnosis of fragile X syndrome, Duchenne or Becker muscular dystrophies, and haemophilia Fluorescence in situ hybridisation is used whereby DNA probes that are complementary to sequences on the $\mathrm{X}$ and $\mathrm{Y}$ chromosomes, as well as a non-sex chromosome sequence as control, are hybridised to the nuclear DNA from a single embryonic cell.

It is apparent that preimplantation genetic diagnosis has the potential for abuse. Indeed it is not allowed in many countries (for example, Germany, Austria, Switzerland, Argentina), and has only recently been allowed in France, whereas in other countries it is virtually unregulated. In the UK it is a procedure regulated by licence from the Human Fertilisation and Embryology Authority (HFEA) under the terms of the Human Fertilisation and Embryology Act (1990). The central aim of the procedure as enshrined in current UK regulations is to prevent the birth of children affected with "very serious, life threatening conditions". The use of preimplantation genetic diagnosis for sex determination outside of this aim is forbidden by the HFEA. As with any new medical technology there is also a grey area in which ethical decisions are particularly controversial. This was highlighted by the use of preimplantation genetic diagnosis to ensure the birth of a baby boy tissue typed so that he could become a donor of haematopoietic stem cells for his sister who suffered from Fanconi's anaemia.' In the UK the HFEA recommended in 2001 that preimplantation tissue typing should only be used when an embryo was being screened for an inherited genetic disorder. However, this decision has recently been under judicial review. The direction such selection procedures could potentially take is illustrated by a recent case in which a child was deliberately conceived using donor insemination by a male with a genetic history of deafness, to be deaf like its lesbian parents. ${ }^{8}$ Ethically it seems wise to focus the use of preimplantation genetic diagnosis on the prevention of births involving lethally destructive genetic mutations, as in present HFEA regulations, and to avoid using the procedure for generating children for utilitarian purposes judged beneficial to their parents or their siblings, but which carry no conceivable benefit to their own welfare. In a market-driven society the "commodification" of babies is a real danger and the intrinsic value of each human individual irrespective of their genetic endowment needs persistent emphasis. Likewise the use of preimplantation genetic diagnosis for the preimplantation selection of embryos on the basis of trivial genetic characteristics without medical implications should be avoided. The emotive term "designer baby" so loved by the media in such discussions is inaccurate as the key human action involved is one of embryo selection not of design. Nevertheless, public unease over excessive levels of selection and control over another person's life are ethically well founded. Such concerns are exacerbated by the small subset of scientists who insist on presenting human genetics in arch reductionist terms.

The postnatal diagnosis of genetic diseases will be greatly facilitated by the sequencing of the human genome, now almost complete. There are already around 1000 documented disease genes out of the approximately 30000 genes in the genome.' Where treatment or a change in lifestyle can ameliorate symptoms there seems every reason to proceed with diagnosis. However, the issues become more complex when, as in Huntington's disease, symptoms may not develop for several decades after the person has received the news that they carry the defective gene. Identification of mutant genes that predispose towards disease but do not guarantee it are likewise difficult to handle, particularly if there are no known environmental changes that will lower the risk. In such cases the right not to know one's genetic constitution may be as important as the right to know. A potential abuse of genetic engineering is to give people genetic information about which they can do nothing, a trend that could encourage genetic fatalism. There is also the continued risk of creating a "genetic underclass" who are less able to obtain life insurance or loans. As Francis Collins, Director of the National Center for Human Genome Research, comments: "Unfortunately there is going to be a gap between our ability to carry out diagnostic work and our ability to intervene therapeutically for a large number of diseases, at least for the next few years. Living in that gap is going to be an uncomfortable experience for all of us .....". ${ }^{10}$

Fortunately the applications of genetic engineering in the treatment of genetic disease is at last yielding some positive results, albeit modest and not without setbacks. In April 2002, after a gene therapy trial that occurred two years previously, French researchers announced that the immune systems of several children with X-linked severe combined immunodeficiency (SCID) were nearly normal. ${ }^{11}$ Out of the 11 children in the trial at the Necker Hospital in Paris, nine were cured. A successful and improved gene transfer protocol for treating SCID patients with adenosine



Figure 1 Multiple uses of genetic engineering; the figure is illustrative only, not exhaustive. White arrows indicate applications that have aroused little ethical controversy whereas black arrows highlight more controversial applications, either actual or proposed. 
deaminase deficiency was also recently reported. ${ }^{12}$ Unfortunately two of the Necker Hospital SCID patients later developed a leukaemia-like condition due to T-cell hyperproliferation, caused by integration of the vector into the LMO-2 gene, mutations which are known to be involved in childhood cancers. The potential risk of such "insertional mutagenesis" events remain a matter for active discussion and may impinge on the 600 gene therapy trials already ongoing worldwide.

The future potential for somatic cell gene therapy remains enormous. The possibility of germ line therapy has also frequently been mooted, but the increasing success of preimplantation genetic diagnosis appears to render this approach unnecessary. Why bother with genetic therapy of an affected early embryo when a non-affected embryo may already be available for implantation?

An important focus for genetic engineering continues to be the diagnosis and healing of human disease. In contrast the suggested use of genetic engineering for so-called "additive" or "enhancement" therapies represents a very different kind of application and the potential for abuse seems high. The term "therapy" is in any case inappropriate as in reality no therapy would be involved, only the aim to enhance certain specified characteristics. Fortunately the complexity of the genome itself represents a natural defence against such interventions. Humans all too frequently aspire to god-like power and wisdom, but the reality of war, the inequitable distribution of resources, and the frequent misuse of science act as constant reminders that our actions do not always live up to our aspirations. The techniques of genetic engineering, if used wisely, can continue to bring enormous benefits to humankind. Arguably those benefits can best be safeguarded within a world view that ensures a high view of the value of the human individual that is independent of the variation in their genetic endowment. ${ }^{13}$

Postgrad Med J 2003;79:249-251

\section{Author's affiliations}

D R Alexander, Chairman of the Molecular Immunology Programme at the Babraham Institute; Fellow of St Edmund's College, Cambridge; and Editor of the journal Science and Christian Belief

Correspondence to: Dr Denis Alexander, Molecular Immunology Programme, Babraham Institute, Babraham, Cambridge CB2 4AT, UK. denis.alexander@bbsrc.ac.uk

\section{REFERENCES}

1 Consortium MGS. Initial sequencing and comparative analysis of the mouse genome. Nature 2002;420:520-62.

2 Huettner CS, Zhang P, Van Etten RA, et al. Reversibility of acute B-cell leukaemia induced by BCR-ABL1. Nat Genet 2000;24:57-60.

3 Schmitt CA, Fridman JS, Yang M, et al. Dissecting p53 tumor suppressor functions in vivo. Cancer Cell 2002; 1:289-98.

4 Yu Y, Bradley A. Engineering chromosomal rearrangements in mice. Nat Rev Genet 2001;2:780-90.

5 Copeland NG, Jenkins NA, Court DL. Recombineering: a powerful new tool for mouse functional genomics. Nat Rev Genet $2001 ; 2: 769-79$.

6 Braude P, Pickering S, Flinter F, Ogilvie CM. Preimplantation genetic diagnosis. Nat Rev Genet 2002;3:941-55

7 Verlinsky Y, Rechitsky S, Schoolcraft W, et al. Preimplantation diagnosis for Fanconi anemia combined with HLA matching. JAMA 2001;285:3130-3

8 Savulescu J. Education and debate: deaf lesbians, "designer disability," and the future of medicine. BN 2002;325:771-3.

9 Jimenez-Sanchez G, Childs B, Valle D. Human disease genes. Nature 2001;409:853-5.

10 Collins F. The human genome project. Science and Christian Belief 1999;11:99-111.

11 Hacein-Bey-Abina S, Le Deist F, Carlier F, et al. Sustained correction of X-linked severe combined immunodeficiency by ex vivo gene combined immunodeficiency by ex vivo gene

12 Aiuti A, Slavin S, Aker M, et al. Correction of ADA-SCID by stem cell gene therapy combined with nonmyeloablative conditioning. Science 2002;296:2410-3.

13 Alexander DR. Rebuilding the matrix - science and faith in the 21 st century. Oxford: Lion, 2001. 